\title{
Femoral Hernia Complications: Unusual Contents
}

\author{
Hamri Asma, Narjis Youssef and Benelkhaiat Ridouan \\ Department of Surgery, Ibn Tofail Hospital, University of Medicine and Pharmacy, Marrakech 40000, Morocco
}

\begin{abstract}
Different contents in the femoral hernia have been reported in the literature, but herniation of the fallopian tube in a femoral hernia is very rare due to its normal anatomical position. CASE Report: A 58 year-old woman, mother of 5 children, she has admitted to surgical ward with a painful lump in the right groin. It had recently increased in size. Clinical examination revealed a $4 \mathrm{~cm} \times 3 \mathrm{~cm}$ tender, irreducible, nonpulsatile mass in the right groin just under the inguinal ligament. There was no cough impulse. A diagnosis of a femoral hernia was made. Exploration of the mass confirmed the diagnosis of the femoral hernia, but sac contained the right fallopian tube. The uterine tube was easily reduced back into the abdomen as it looked healthy and the hernia defect was repaired with Mac Vay technique. The patient made good recovery. CONCLUSION: This case is educational as it highlights the importance of managing women with femoral masses with care.
\end{abstract}

Key words: Fallopian tube, femoral hernia.

\section{Background}

Herniation of the uterine tube is rare due to its anatomical position. If it happens, its status should be properly checked as the treatment depends on its viability. Considering the rarity of this event, we present a new case of a femoral hernia containing incarcerated fallopian tube in old woman

\section{Case Report}

A 58 year-old woman, mother of 5 children, she has admitted to surgical ward with a painful lump in the right groin. It had recently increased in size. Clinical examination revealed a $4 \mathrm{~cm} \times 3 \mathrm{~cm}$ tender, irreducible, nonpulsatile mass in the right groin just under the inguinal ligament. There was no cough impulse. A diagnosis of a femoral hernia was made. Exploration of the mass confirmed the diagnosis of the femoral hernia, but sac contained the right fallopian tube (Fig. 1). The uterine tube was easily reduced back into the abdomen as it looked healthy and the hernia defect was repaired with Mac Vay technique. The patient made good recovery.

Corresponding author: Benelkhaiat Ridouan, Ph.D., research field: surgery.

\section{Discussion}

Femoral hernias are more common in women and lead to a substantial higher rate for an emergency operation, due to strangulation. Incarcerated femoral hernia with fallopian tube as a content is an extremely rare condition [1]. The diagnosis of a femoral hernia is usually clinical. Most patients present as an emergency. A typical femoral hernia presents as a tender, nonreducible swelling with no cough impulse and is situated below and lateral to the pubic tubercle. On exploration, it often contains only omentum, or may contain a knuckle of bowel known as Richter's hernia. The differential diagnoses include inguinal hernia, lipoma, saphena varix, enlarged lymph nodes, femoral artery aneurysm, sarcoma, obturator hernia, psoas abscess, psoas bursa, and in males, ectopic testis.

Inguinal herniation of the adnexa is rare and is generally found in children with associated congenital abnormalities of the genital tract. In his analysis of 1,950 patients with inguinal hernias, Gurer et al. [2] found that most of his patients were adults with no genital abnormalities. The incidence of inguinal hernias containing ovary and fallopian tube in his study was $2.9 \%$. Herniation of the fallopian tube in a femoral hernia however is very rare due to its normal 


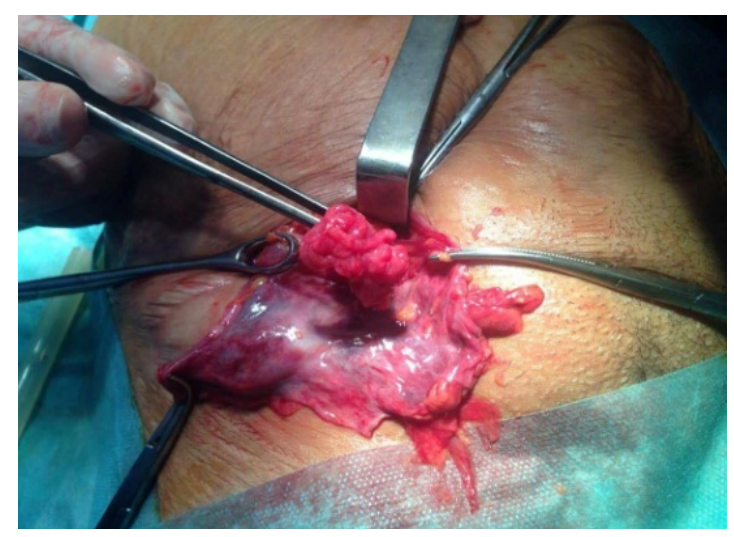

Fig. 1 Fallopian tube in the explored femoral hernia.

anatomical position as it lies at a lower level than the femoral ring [3]. In infants, the fallopian tube is near the internal inguinal ring and can more readily pass through this structure than later in life, due to the closure of the canal of Nuck. Due to its deep pelvic position, it is very difficult for the uterine tube to herniate in adults unless there is a sudden anomalous movement when the body is forcibly inclined towards the inguinal region, for example, or a pathological condition of the internal genital organs such as a uterine fibroid [4].

Patients with herniation of the fallopian tube might present with unusual symptoms which vary from intermittent attacks of abdominal pains, irreducibility and strangulation, increased hernia size during muscular effort, to those which resemble symptoms of ileus. Sometimes, a cord-like body in the sac leading to the hernial opening might be palpated. A vaginal bimanual examination help diagnose such cases by findings a deviation of the uterus towards the side of the hernia; for instance, the uterus being drawn over and inclined towards the side on which the hernia was situated, or the uterus is in a position of marked anteflexion and seemingly fixed [4]. If the tube is healthy on exploring the hernial sac, it should be reduced into the pelvic cavity. Otherwise, it should be removed if its viability is in doubt, gangrenous, or profound pathological changes have occurred [5]. In children, the peritoneal attachment of the fallopian tube and the ovary to the posterior wall has to be divided before reducing them if they are situated in the canal of Nuck as they are more or less fixed at this position [6].

\section{Conclusion}

Despite its rarity, one should be aware, especially when treating female patients presenting with clinically strangulated femoral hernias.

\section{References}

[1] Atmatzidis, S., Chatzimavroudis, G., Dragoumois, D., and Atmadzidis, K. 2010. "Incarcerated Femoral Hernia Containing Ipsilateral Fallopian Tube.” Case Reports in Medicines (2010): 741915.

[2] Gurer, A., Ozdogan, M., Ozlem, N., Yildirim, A., Kulacoglu, H., and Aydin, R. 2006. "Uncommon Content in Groin Hernia Sac.” Hernia 2 (10): 152-5.

[3] King, R. 1987. “Congenital, Strangulated Femoral Hernia in an Infant Aged Six Months Containing the Ovary and Fallopian Tube.” British Journal of Clinical Practice 7 (32): 209-10.

[4] Morf, P. F. 1901. "Hernia of the Fallopian Tube without Hernia of the Ovary.” Annals of Surgery 3 (33): 247-68.

[5] Alzaraa, A. 2011. "Unusual Contents of the Femoral Hernia.” ISRN Obstetrics and Gynecology 2 (2011): 717924.

[6] Carmichael, E. S. 1906. "Hernia of the Uterine Adnexa, with a Personal Experience of Seven Cases.” Journal of Obstetrics \& Gynaecology 10 (1): 16-21. 